\title{
Ultra Artificial Intelligence (UAI): Redefining AI for New Research Dimension
}

\section{Md. Shaikh S}

Institute of Management and Science (IMS), Sakegaon-Bhusawal, Maharashtra, India

*Corresponding author: Md. Sadique Shaikh, Institute of Management and Science (IMS), Sakegaon-Bhusawal, Maharashtra, India, Tel: 093264 61497; E-mail: sids_nsk@rediffmail.com

Received date: Mar 15, 2017; Accepted date: Apr 13, 2017; Published date: Apr 21, 2017

Copyright: () 2017 Md. Shaikh S. This is an open-access article distributed under the terms of the Creative Commons Attribution License, which permits unrestricted use, distribution, and reproduction in any medium, provided the original author and source are credited.

\section{Introduction}

Neuroscience is most important subject which gives information about world most complex biological intelligence system i.e., brain, how reasoning, knowledge and creativity possible for it, give data and information of brain functioning. When strong and successful results, theories and, practices achieved in it, human race start to think, why not we mimic our brain. And the result of thinking, dreaming and engineering to obtain it, in the result term "Artificial Intelligence". We much familiar with the term AI, it is science and Engineering to design electronic logic which function like God made brain (natural intelligence) on silicon wafer which is man-made called Artificial Intelligence. Researchers, engineers and practitioners got succeed to design and implement two types of AI i.e., Hardware AI and software $\mathrm{AI}$ with two different nature i.e., weak $\mathrm{AI}$ and strong $\mathrm{AI}$, and humanoid and Android examples of strong hardware AI and software AI respectively. We are the civilization which living in hi-tech IT world and WWW and due to computing (Specially Advanced Micro/Nano Processors) and Artificial Neural Network (ANN) Engineering with modelling, simulation and Advance chip fabrication as sell as programming languages, human race exit from weak $\mathrm{AI}$ and jump into strong AI and result of it ASIMO (Advanced Step In Mobility) first its kind of successful Humanoid Robot. Came with various version of it and manufactured by HONDA. ASIMO has ability like speech recognition, image processing, human-like movement processing, voice generation and self-programming algorithm, but researcher, practitioner and engineers still fail to engineer love, emotions, feelings, ability of wise self-decision-making and to distinguish good or bad activities to do and moving for it. But here my objective is different to discuss with the help of this Science Fiction article with term "Ultra Artificial Intelligence (UAI)". Since Darwin's years, Human started to use their brains potentially, like logical and arithmetic, reasoning, creativity, gestures, languages and their means encoding and decoding to response each other, but perhaps human being start to brain for knowledge, reasoning, creation and intelligence development. At present unfortunately we using it and how it functioning fail to know; Today's psychologist, neurologists and practitioners show several research results but still uncertainty maintained from that era to this era, how human brain work; And up to what level we knew it we convert it artificially on silicon chip using ICs fabrication technology like ULSI and Nanotechnology. Today's researcher even able to design and implement ICs which work similar like human brain with the success to convert biological Neuron mesh and engineering using tools ANN and advanced computer languages and algorithms called "Bioelectronic" in short "Bionic" Brain [1-3]. The term "Bionic Brain" still matter of debate and belong to "Sci-fi". My traced models would be possibility after the successful design and development of "Bionic
Brain". When this term change to reality, my work will become a serious and interesting matter to start thinking called "UAI" (Figure 1).

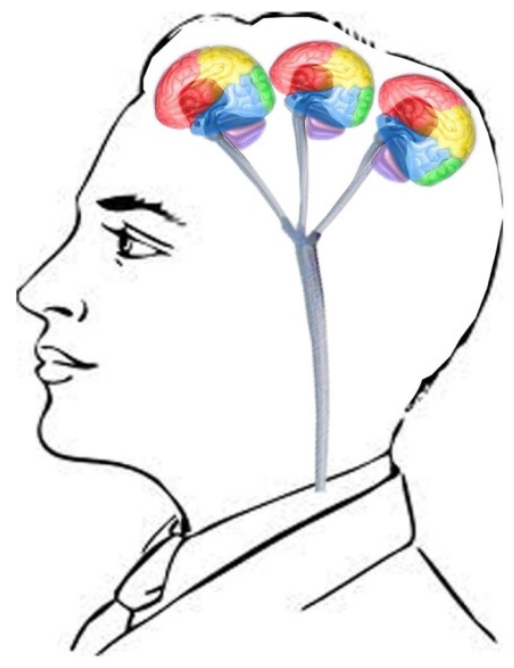

Figure 1: Bionic Brain.

My proposed research work is completely hypothetical and science fiction, but big direction for lateral thinking to all researchers, who are interested to work in AI domain. To understand term UAI, Now I would like to back again on human brain example. Think if man or woman having more than one brain in his/her skull, and all these brains connected through single nervous system and all brain function for single person so what would be level of his/her knowledge, realizing, creation and intelligence when all brains think simultaneously. But the reality is above said statement seems to be impossible and sound very stupid, of course not possible in case of human biological brain which is always single and God given and if God decided to give multiple biological brain to single person, then term would be "Ultra Natural Intelligence (UNI)" but it highly impossible in case of living organism, even by any brain surgery etc. but If we apply same statement in non-living organism or electronics or Bionic brain context, than it would be completely valid and possible on one day. "Bionic Brains" is AI and by proper engineering it seems to be possible one could cascade multiple Bionic Brain and generate inter-link among them using single system bus like Bionic Brain-1, BB-2, ......BB- $\mathrm{n}$, where $\mathrm{n}$ is desired number or last count when such system using multiple Bionic Brain become possible to design and implement where all BBs function simultaneously term could say," Ultra Artificial Intelligence, UAI". If we talk about "Bionic Brains", they are not only Artificial Intelligence (AI), but Super Artificial Intelligence (SAI), already themselves, and multiple BBs with SAI integrated that 
Page 2 of 3

develop UAI. Hence, I wanted to broad further this concept with definition and models [4-7].

\section{Definition}

The term can be defined as "Ultra Artificial Intelligence (UAI) system design and development is set of all required Super Artificial Intelligence (SAIs) with integration and co-functioning with each other for Ultra High data processing within a very small fraction of second, where general AIs consume minute to do it."

\section{UAI Routing Model}

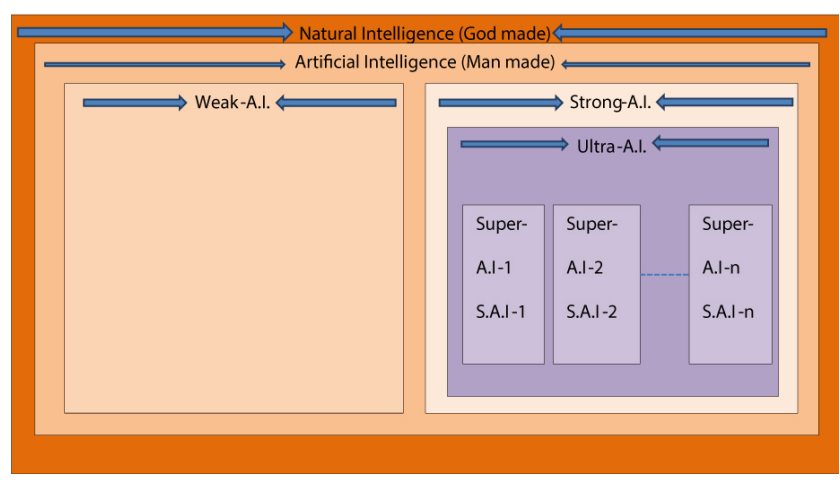

Figure 2: UAI Routing Model.

Above Figure 2 shows exhibit display conceptualized UAI Routing Model. This model show intelligence penetration and extraction at each level to finally achieved UAI. The concept AI is extracted and engineered from Natural Intelligence (NI) which God gift to human being called biological brain, that function on neurons processing. This NI with study of brain, neuroscience and ability of reasoning, logical, arithmetic, creativity and finally after studied transfer it on silicon wafer artificially. AI domain further has two nature in it i.e., weak AI and strong AI, where weak AI means small machine intelligence engineered electronically on chip or any small program/software/ procedure for recursive repetitive/iterative task or function and of course not our interest to discuss, But another domain strong AI is real AI engineering need for Humanoid Robotics. Strong AI responsible for machine vision, voice recognition, template matching, thinking machine, self-programming and multi-functioning. As it clearly showcase in model to construct UAI, we need multiple cascaded SAI, where single SAI build using thousands of strong AI engineering efforts. Where single UAI has number of requisite super AI for each intelligence individually from first SAI to nth one, but all functioning with co-ordination like mechanical intelligence, structural intelligence, self-programming and decision-making intelligence and so on, we will discuss in next coming model. Hence from this model I again want to prove definition UAI is Superset of all Strong Super AI which are integrated and co-functional [8-10].

\section{Single - UAIBB (Ultra Artificial Intelligence Bionic Brain) Model}

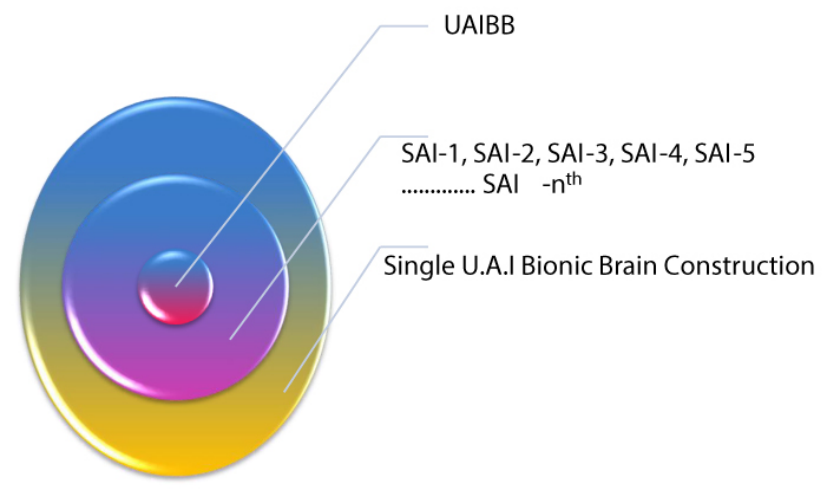

Figure 3: Single - UAIBB (Ultra Artificial Intelligence Bionic Brain) Model.

Above proposed model is exact representation of UAI definition and isolately support in detail to UAI Routing Model, where strong AI need to build UAI discussed with penetration [11] (Figure 3).

In UAIBB model, it is clearly shown to engineer and implement effective UAI, very first we need to complete engineering success in strong AI, then Super AI (SAI) and when we got success in SAI, we would be newer to think and design UAI, because after the success to design and develop Super AI, where super AI itself biggest collection of all types of strong AI, but still if we wish to create any machine intelligence which is not only human being, but also thousands of time faster in processing, we need to think about this grand design seriously. If we got success to do so, means we got success to design UAIBB using UAI (set of SAIs), as displayed from SAI-1, SAI-2, SAI-n. Hence natural intelligence of human brain, one day could engineer Ultra Artificial Intelligence several time advance of it [12].

\section{Bionic Brain Engineering Model}

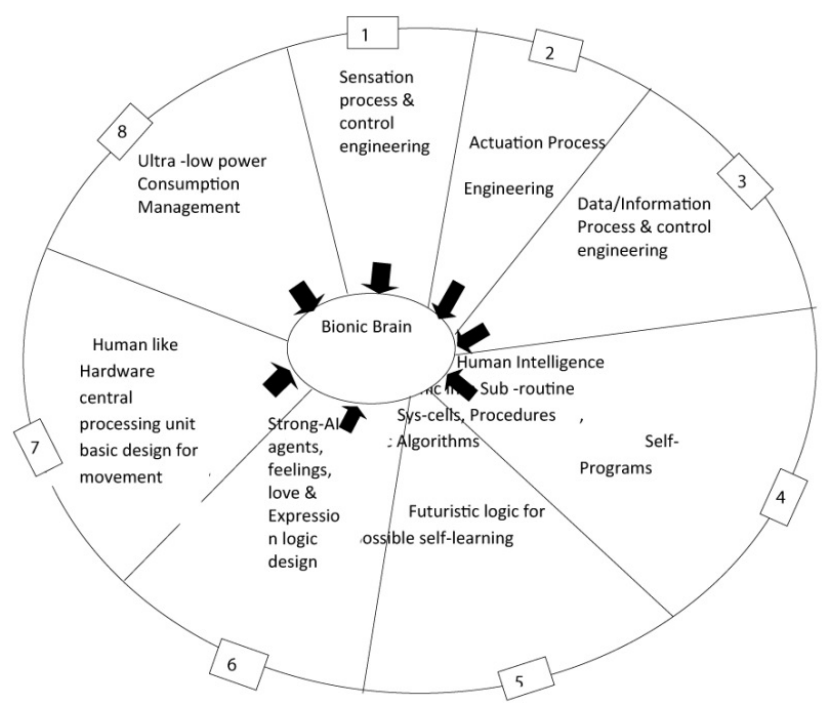

Figure 4: Bionic Brain Engineering Model.

This is the last model I traced with present and future considerations in Humanoid engineering with special preference to 
Page 3 of 3

"Bionic Brain" hence named it "Bionic Brain Engineering Model (BBEM)". This model is further segmented into 8 segments of Bionic Brain Engineering like 'sensation process and control engineering' which reach environment stimuli's up to Bionic Brain of Humanoid i.e., how to sense and process input signal from human and environment through sensor using intelligence software supports. Second is 'Actuation Process and control engineering', for output human like response from Robot after sensation and processing input signal, in this engineering segment also need to well synchronized intelligence software with physical body i.e., hardware parts of Humanoid Robot like motors, joints, pneumatic units and control, storage, logical processor, buffers, servo motors and so on. Next important engineering is 'Data information processes and control engineering' i.e., how robots accept and process voice signal, text, images, smell, touch and likewise several signals as well as identify and control them during processing. Next is 'Human Intelligence mimicking 'into sub-routine, sys-calls, procedures, genetic Algorithms, Neural Network, Neural Schema's and so on. Next and very important domain of Bionic brain engineering for Humanoid is 'self-learning programs and futuristic logic for possible self-learning. Simultaneously algorithm forth engineering domain fifth domain must have to interfere with forth one i.e., 'Strong - AI 'and Strong - AI agents, logic designing and programming for feelings, love, emotions, expressions, desires etc. One very important engineering aspect of Humanoid is also how could Human-like central processing control hardware possible to develop look like human body and skill and movement like human body. After this next engineering approach is ultra-low power consumption management i.e., designing of small, highly dense, durable, reliable with high density of storage, memories, buffers, storage required, generally where energy sources are inbuilt and rechargeable to give mobility and autonomy to Human Robotics $[13,14]$ (Figure 4).

\section{Conclusion}

Ultra Artificial Intelligence would be one of the major discoveries to 2025 and might be able to engineer and implement up to 2040. Successful designing and development of UAI will brings lot of technological and computer/electronic intelligence advances in this world and Humanoid Robots engineered using UAIBB seems to be like Alien in this world. These would be UAI Robots exactly look like human being and will disperse in our civilization, they all would be self-programmable machines not only having intelligence, but also decision-making, emotions, feelings, sex, violence, revenge, ego, sympathy, pain and likewise factors. It's only Utopia about UAI based Humanoid. They would be friends of human civilization, it might has risk like they can make their own humanoid civilization and oppose to human civilization and treat them as an enemy of their and we people unable to control and defence from those advance machine intelligence which are thousand times stronger than brain and might try to destroy human civilization or rule out on world. It might be possible our made up machine will become a reason of our destruction in this world. But this is only the hypothesis of future situation may be different and they may good friends of human being with proper control and selfdestruction programming of humanoid or designer develop any computer humanoid virus, which may become a cause of malfunctioning and destruction of Humanoid if they become dangers and hazardous for human civilization. This would be miracle of ordinary natural intelligence (Brain) to design and develop extraordinary Artificial Intelligence (Bionic Brain). It is also possible some from us create some anti-program to use Humanoid against of Human civilization as enemy instead of servants or friends in the form of virus.

\section{References}

1. Aarup M, Arancli MM, Paned Y, Stader, Stokes 1 09941_CIPTIMLIMA1V: A inosolodge Lowed planning and for spacecraft AlV. In: Fox M, Zwcben M, editors. Knowledge Based Scheduling. Morgan Kauf-mann.

2. Abney S (2007) Semisuperpised Learning for Computational Linguistics. CRC Pins

3. Abramson B, Yang M (1989) Divide and torquer under global constraints: A solution to the N-g Linens problem. I. Parallel mul Dintributea COPE paring, 6121, env) 662 .

4. Acalioptas D (2009) Random satisfiability. In: Siam A, Heige M, Assl Marren H, Walsh TL, editors. A Handbook of Sonsfirreildy 105 Press.

5. Achlloptas D, Bernie P, Molloy M (2004) Exponential bounds for DPLL below die Liy threshold. In: SODA-64.

6. Bacchus R, Grove A, Halpern IY, Koller 0.119'921. From satisfies to beliefs. In: AMMO, pp: 602-608.

7. Bacchus F, van Beek P (1998) On the conversion between non-binary and binary constraint sans-fucuimo gi olden. In: AAAL4R, pp: 311-315.

8. Bacchus F, tin Rim P (1995) Dynamic vain-able ordering in CS9s. In: CP-95, pp: 258-275.

9. Bachmann PGH (1894) Die analytische Zahtem theorie BG Teal: user, Leiptig.

10. Chamlak E (1996) Tern-bank grammars. In: AAV.96: 1031-1036.

11. Chamlak E (1997) Statistical parsing with a context-free grammar and word statistics. In: AAA!. 97: 598-6d3.

12. Charnlak E, Goldman RI (1992) A Bayesian model of plan recognition. ALI 64411, pp: 53-79.

13. Charialak E, McDermott D (1985) Intraday-lion to Arnficial Intelligence. Addison-Wesley. Chandra E, Riesbtol C, McDermott D, i91471 Pre,ram. Ming (2nd edn). Lawrence Erlbaarn Associates.

14. Chamialt E (1991) Bayesian networks without /CATE. AlMug, EJ(d), pp: $55-63$. 